\title{
Studies of vector boson+jet production with ATLAS
}

\author{
Eric JANSEN* \\ On behalf of the ATLAS collaboration \\ University College London (UK) \\ E-mail: eric.jansen@cern.ch
}

\begin{abstract}
The production of vector bosons in association with jets has been studied in proton-proton collisions at $\sqrt{s}=7 \mathrm{TeV}$ with the ATLAS detector at the LHC. Cross sections have been determined differentially in several kinematic variables and up to high jet multiplicities. For the exclusive 1 jet case also the ratio of the $W+1$ jet to the $Z+1$ jet cross section has been measured. The results are compared to the latest fixed-order predictions at NLO and to predictions of generators that use LO or NLO matrix elements supplemented by parton showers. Good agreement is observed between data and most predictions. However, for inclusive quantities such as the scalar $p_{\mathrm{T}}$ sum $H_{T}$, the results demonstrate the need to include higher multiplicity matrix elements in fixed-order NLO calculations.
\end{abstract}

XXI International Workshop on Deep-Inelastic Scattering and Related Subjects 22-26 April, 2013

Marseilles, France

\footnotetext{
*Speaker.
} 


\section{Introduction}

The production of vector bosons in association with jets provides an important test of perturbative QCD (pQCD). Additionally, events with this final state are backgrounds to studies of Standard Model (SM) processes, Higgs measurements, and searches for new physics beyond the SM. Cross sections for $W$ and $Z$ production in association with jets have been measured differentially in several kinematic variables and up to high jet multiplicities using the ATLAS detector [1] at the LHC. Also the ratio of exclusively produced $W+1$ jet to $Z+1$ jet processes has been calculated, which benefits from the cancellation of many theoretical and experimental uncertainties. The results are corrected to the particle level and compared to fixed-order predictions at next-to-leading order (NLO) in pQCD, and to predictions of Monte Carlo (MC) generators that use leading-order (LO) or NLO matrix elements supplemented by parton showers.

\section{2. $W+$ jets}

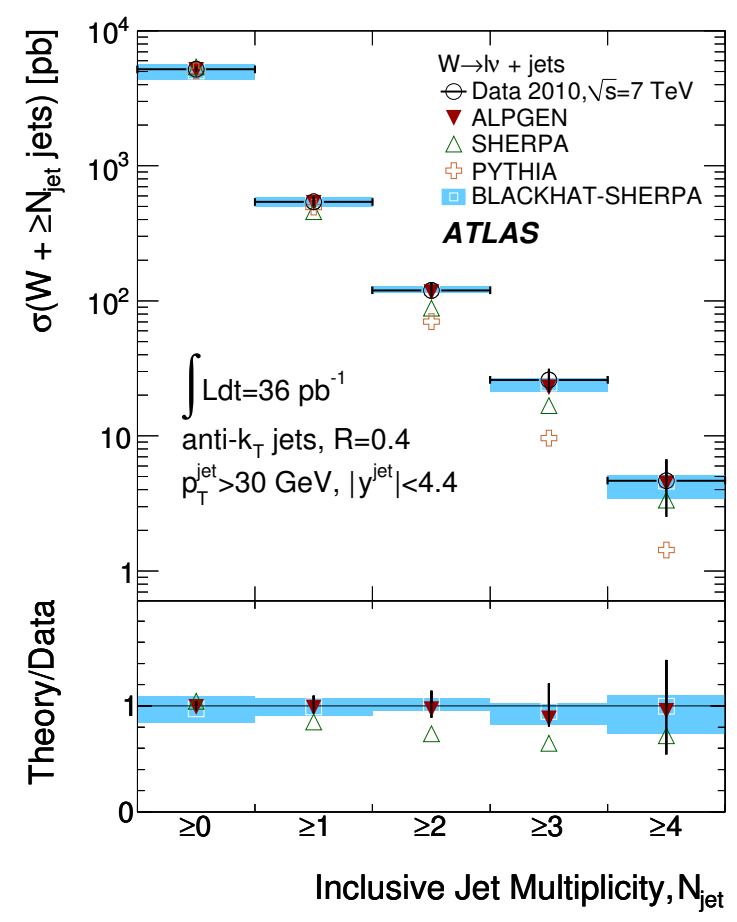

(a) Inclusive multiplicity



(b) Inclusive multiplicity ratio

Figure 1: Production cross section for a $W$ boson in association with jets, as a function of inclusive jet multiplicity and as a function of the ratio of inclusive multiplicities [2].

The $W+$ jets measurement [2] uses $36 \mathrm{pb}^{-1}$ of data collected in 2010. Leptonically decaying $W$ bosons are selected by requiring one lepton $(e, \mu)$ with $p_{\mathrm{T}}^{\ell}>20 \mathrm{GeV}$, a missing energy of $E_{\mathrm{T}}^{\text {miss }}>25 \mathrm{GeV}$, and a $W$ transverse mass ${ }^{1}$ of $m_{\mathrm{T}}>40 \mathrm{GeV}$. Jets are reconstructed using the anti- $k_{t}$

\footnotetext{
${ }^{1}$ The transverse mass is defined as $m_{\mathrm{T}}=\sqrt{2 p_{\mathrm{T}}^{\ell} p_{\mathrm{T}}^{\nu}\left(1-\cos \left(\Delta \phi^{\ell v}\right)\right)}$
} 
algorithm with a radius parameter of $R=0.4$. Their transverse momentum is required to be $p_{\mathrm{T}}^{\text {jet }}>$ $30 \mathrm{GeV}$.

After this selection, the dominant background processes are QCD multijet events, $W \rightarrow \tau \nu$, $Z \rightarrow \ell \ell$ and leptonic $t \bar{t}$ decays. The multijet background consists of light flavour jets that pass the electron selection, and leptonic decays of bottom and charm hadrons. The mechanism by which jets fake leptons is difficult to simulate, therefore the multijet background is estimated using a control region in data. The lepton quality criteria for this control region are reversed, leading to a sample dominated by fake leptons. This sample is used to extract the shape of the $E_{\mathrm{T}}^{\mathrm{miss}}$ distribution for multijet events. A second template, describing the signal and the other backgrounds is obtained from simulation. The background normalisation is determined by fitting the two templates to the $E_{\mathrm{T}}^{\text {miss }}$ distribution in data. This fit is performed separately for each exclusive jet multiplicity.

The resulting cross sections are compared to the LO multiparton plus parton shower predictions of the ALPGEN and SHERPA generators, and to the NLO matrix element calculations obtained using BLACKHAT-SHERPA. Figure 1 shows the $W+$ jets cross section as a function of the inclusive jet multiplicity, and as a ratio of the cross section for different inclusive multiplicities. The data shows good agreement with ALPGEN and BLACKHAT-SHERPA. The agreement with SHERPA is slightly worse.

\section{Z+jets}

The $Z+$ jets cross section has been measured using $4.6 \mathrm{fb}^{-1}$ of data at $\sqrt{s}=7 \mathrm{TeV}$ [3]. The selection proceeds largely analogous to the $W+$ jets case. The $Z$ bosons are selected by requiring two same-flavour opposite-sign leptons, with an invariant mass in the window $66<m^{\ell \ell}<116 \mathrm{GeV}$. The background is dominated by $t \bar{t}$ and for low multiplicities also by diboson and QCD multijet production. Background estimates are obtained using the same methods as in the $W+$ jets cross section, with the exception of $t \bar{t}$. The $t \bar{t}$ component is estimated by selecting opposite-flavour opposite-sign lepton pairs, using MC simulation to extrapolate this to a prediction for the sameflavour background.

Figure 2a shows the cross section for inclusive jet multiplicities up to seven jets. The data are compared with the latest BLACKHAT-SHERPA $Z+3 / 4$ jet predictions $[4,5]$ and with the predictions of ALPGEN and SHERPA. The data are found to be consistent with all of these predictions. The measured cross section is also compared to the prediction of MC@NLO. In contrast to ALPGEN and SHERPA, MC@NLO generates the Drell-Yan process at NLO precision (including one additional parton), while all higher multiplicities are generated by the parton shower. The MC@ NLO parton shower underestimates the observed rate for additional jet emission by a factor of two, leading to a large deficit with respect to data at high jet multiplicities.

Quantities based on inclusive $p_{\mathrm{T}}$ sums are often used in searches for the decays of heavy particles. One such quantity is $H_{\mathrm{T}}$, defined as $H_{\mathrm{T}}=E_{\mathrm{T}}^{\text {miss }}+\sum_{\ell \text {,jet }}\left|p_{\mathrm{T}}\right|$, where the sum runs over all leptons and jets in the final state. Figure $2 \mathrm{~b}$ shows the differential cross section as a function of $H_{\mathrm{T}}$, normalised to the $Z \rightarrow \ell \ell$ cross section. The $H_{\mathrm{T}}$ spectrum predicted by ALPGEN is slightly too hard, while SHERPA shows a 10-15\% offset with respect to data. The BLACKHAT-SHERPA prediction, on the other hand, deviates increasingly from data for larger values of $H_{\mathrm{T}}$. This discrepancy is attributed to missing higher jet multiplicities in the fixed-order calculations. A NLO $n$ jet prediction 


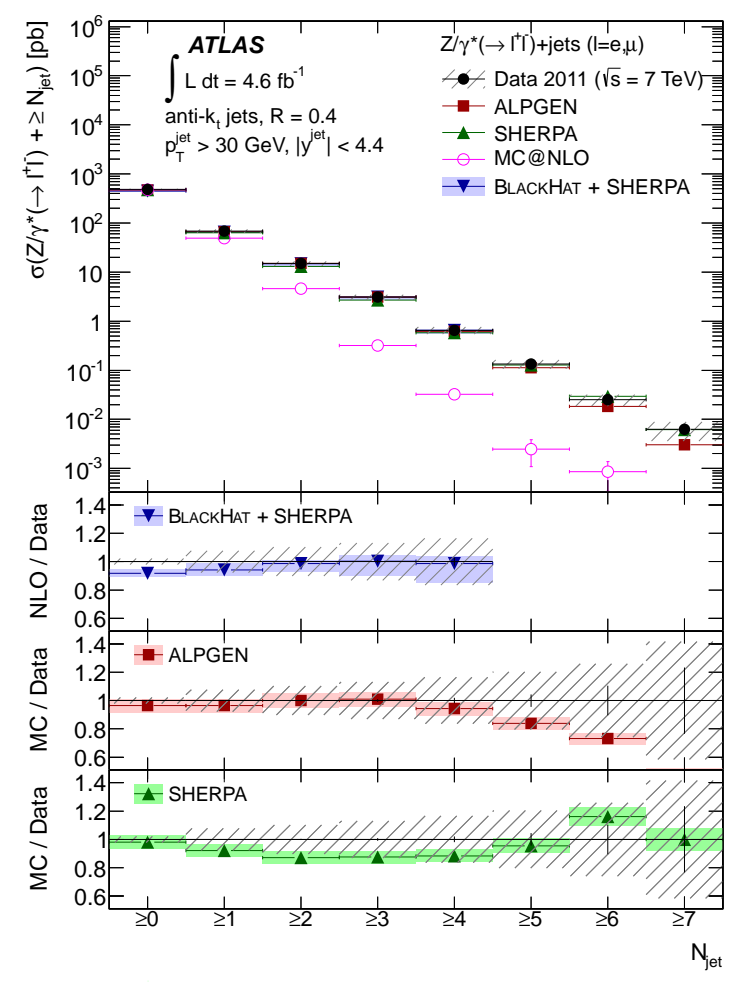

(a) Inclusive jet multiplicity

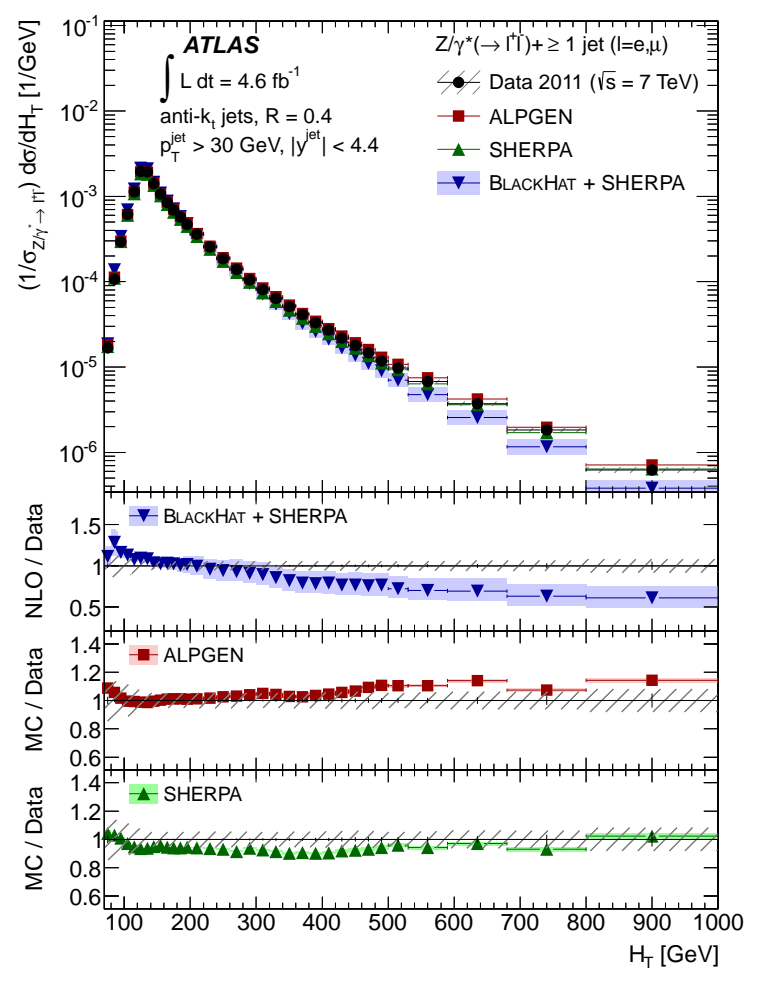

(b) $H_{\mathrm{T}}$

Figure 2: The $Z+$ jets production cross section as a function of inclusive jet multiplicity and the scalar transverse momentum sum $H_{\mathrm{T}}[3]$.

produces events with $n$ or $n+1$ partons. However, for some inclusive quantities the higher multiplicities do have a significant impact, as is the case with $H_{\mathrm{T}}$. Reference [6] introduces the exclusive sums technique, which attempts to mitigate the problem by adding NLO predictions for different multiplicities together. In this way the $n+1$ jet component is removed from the $n$ jet prediction and replaced by the full $n+1$ jet prediction. The exclusive sum of the 1 jet and the $\geq 2$ jets prediction is consistent with the observed $H_{\mathrm{T}}$ spectra. The same behaviour has been observed previously in $W+$ jets [2].

Another observable that was studied is the ratio between subsequent exclusive jet multiplicities, $R_{(n+1) / n}$. This ratio is expected to be described by a combination of two benchmark patterns: staircase scaling, with $R_{(n+1) / n}$ constant; and Poisson scaling, with $R_{(n+1) / n}$ inversely proportional to $n$ [7]. At high multiplicities the non-abelian nature of QCD final state radiation predicts a flat exclusive jet multiplicity ratio, while for low multiplicity the ratio is expected to be flat due to the combined effect of a Poisson-distributed multiplicity and parton density suppression. The underlying Poisson scaling is expected to emerge after introducing a large scale difference between the core process and the $p_{\mathrm{T}}$ of the second leading jet. Two measurements of $R_{(n+1) / n}$ are performed to test this: one using the standard selection, shown in Fig. 3a; and the other requiring a leading jet momentum of $p_{\mathrm{T}}>150 \mathrm{GeV}$, shown in Fig. 3b. The two scaling patterns are observed in data and are accurately reproduced by all MC generators. 


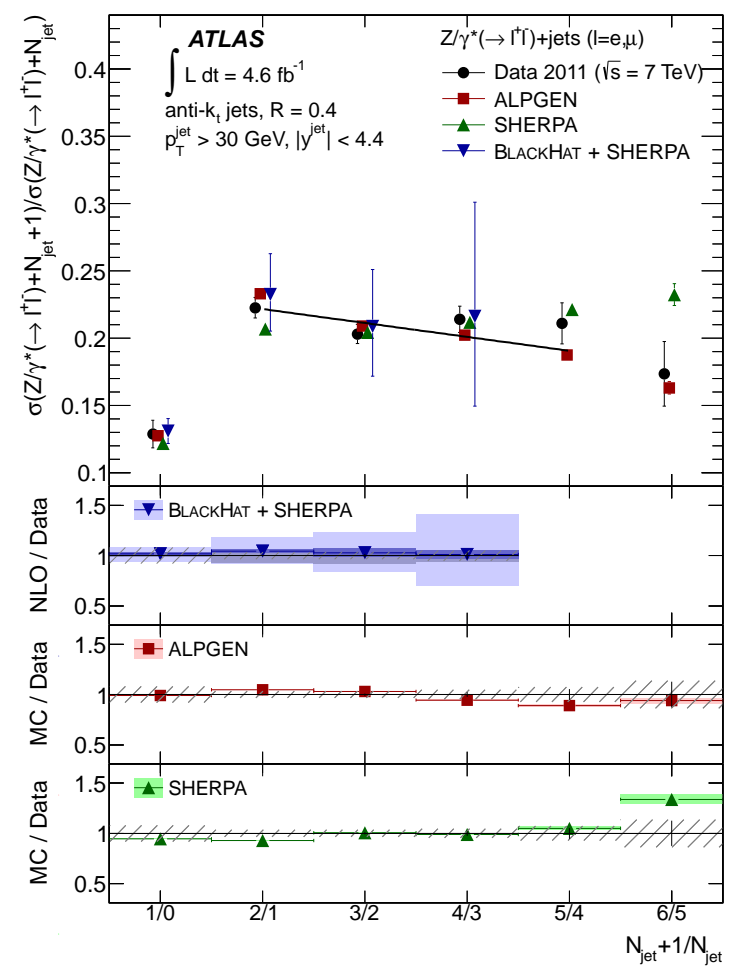

(a) $p_{\mathrm{T}}^{\text {jet }}>30 \mathrm{GeV}$

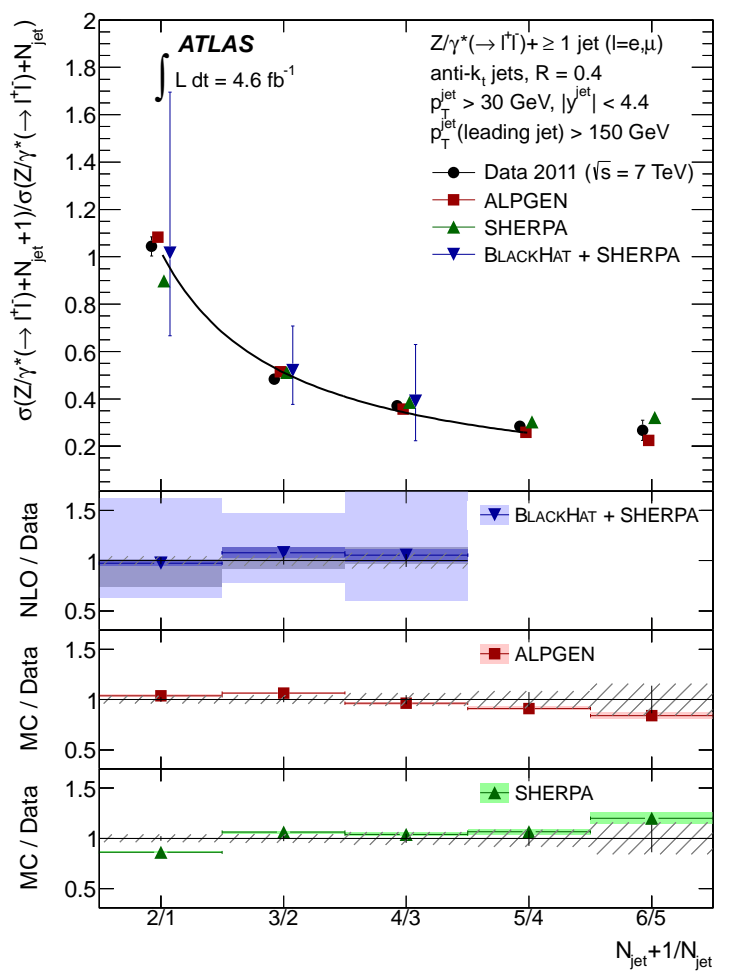

(b) $p_{\mathrm{T}}^{\text {jet }}($ leading jet $)>150 \mathrm{GeV}$

Figure 3: The $Z+j$ ets exclusive jet ratio in the absence of major kinematic cuts and when requiring a high $p_{\mathrm{T}}$ leading jet [3]. The different uncertainties shown for BLACKHAT-SHERPA indicate different treatments of the scale variation: no scale variation (dark shaded), scale variation correlated between bins (medium shaded) and uncorrelated between bins (light shaded).

\section{4. $W+1$ jet $/ Z+1$ jet ratio}

For the exclusive 1 jet case, ATLAS has measured the ratio between the $W+1$ jet and $Z+1$ jet cross section using an integrated luminosity of $33 \mathrm{pb}^{-1}$ [8]. Due to the cancellation of theoretical and experimental uncertainties, this ratio provides a high precision test of the Standard Model. The selection criteria are similar to those used for the $W+$ jets and $Z+$ jets measurements, with the exception of $\left|\eta^{\text {jet }}\right|<2.8$ and the $Z$ mass window which is reduced to $71<m^{\ell \ell}<111 \mathrm{GeV}$.

Figure $4 \mathrm{a}$ shows the ratio of cross sections as a function of the jet $p_{\mathrm{T}}$ threshold for the fiducial volume of $\left|\eta^{\ell}\right|<2.5$ and $p_{\mathrm{T}}^{\ell}>20 \mathrm{GeV}$. The systematic uncertainty is dominated by the boson reconstruction and ranges from $4 \%$ at low jet $p_{\mathrm{T}}$ to $15 \%$ at the highest jet $p_{\mathrm{T}}$ threshold. Above $50 \mathrm{GeV}$ the total uncertainty is dominated by the statistical uncertainty. The ratio has also been extrapolated to the full boson phase space, which is shown in Fig. 4b. The measured ratios are compared to the LO predictions of ALPGEN and PYTHIA and to the NLO prediction obtained using MCFM. Good agreement is observed over the full threshold range. 


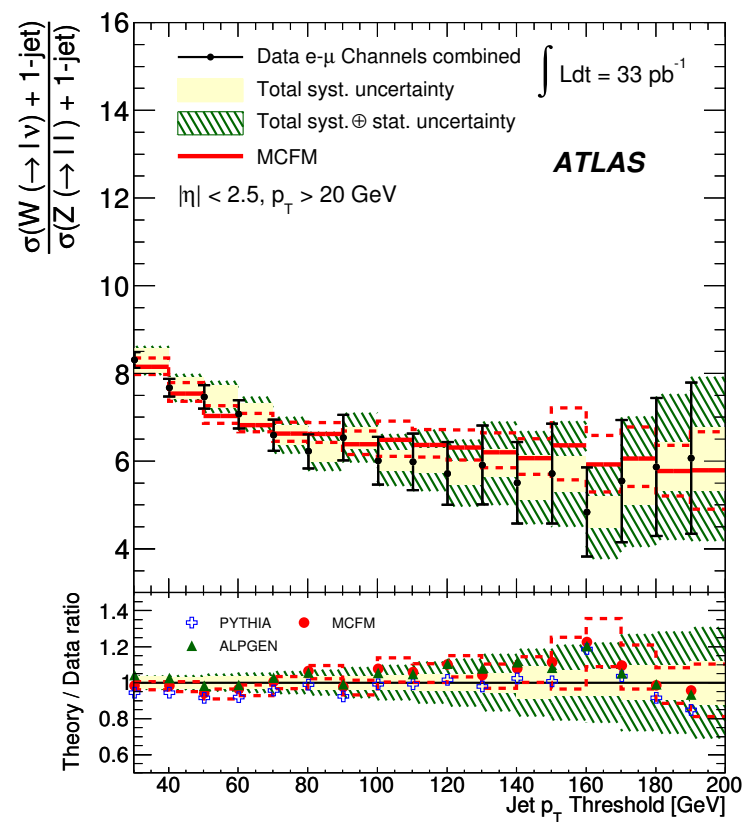

(a) Fiducial volume



(b) Full boson phase space

Figure 4: Ratio of the $W+1$ jet to the $Z+1$ jet cross section as a function of the jet $p_{\mathrm{T}}$ threshold. [8]

\section{Conclusions}

Cross sections for $W$ and $Z$ production in association with jets have been measured differentially in several kinematic variables and up to multiplicities of $\geq 4$ and $\geq 7$ jets respectively. Also the ratio of the $W+1$ jet to the $Z+1$ jet cross section has been calculated. The results have been compared to the latest fixed-order NLO predictions and to predictions of generators using LO or NLO matrix elements supplemented by parton showers. The results are generally found to be in good agreement with the theory predictions. However, the differences observed for inclusive quantities such as $H_{\mathrm{T}}$ demonstrate the need to include higher multiplicity matrix elements in fixed-order NLO calculations.

\section{References}

[1] ATLAS Collaboration, JINST 3 (2008) S08003.

[2] ATLAS Collaboration, Phys.Rev. D85 (2012) 092002 [arXiv: 1201.1276].

[3] ATLAS Collaboration, Submitted to JHEP [arXiv: 1304 . 7098].

[4] C.F. Berger et al., Phys.Rev. D82 (2010) 074002 [arXiv: 1004 . 1659].

[5] H. Ita et al., Phys.Rev. D85 (2012) 031501 [arXiv: 1108 .2229].

[6] J. Alcaraz Maestre et al., [arXiv:1203:6803].

[7] E. Gerwick et al., JHEP 1210162 (2012) [arXiv: 1208 . 3676].

[8] ATLAS Collaboration, Phys.Lett. B708 (2012) 221-240 [arXiv: 1108 . 4908]. 